\title{
Evaluation of the OhmMapper Instrument for Soil Moisture Measurement
}

\author{
Jeffrey P. Walker* and Paul R. Houser
}

\begin{abstract}
An instrument for making rapid measurements of the soil moisture content in the root zone is an essential tool for many applications, including understanding of soil water dynamics, evaluation of agriculture water stress, and validation of soil moisture modeling. Studies have shown that electrical resistance measurements may be used to infer soil moisture content under special circumstances. In this paper, electrical resistivity (resistance multiplied by a geometric factor) measurements of the soil by the Geometrics Inc. OhmMapper instrument are compared with point measurements of soil moisture to a depth of $70 \mathrm{~cm}$. It was found that the OhmMapper resistivity measurements could be used to infer soil moisture content with a coefficient of determination as high as 0.34 when a simple power law relationship was used. A more sophisticated analysis of the resistivity measurements could potentially lead to a greater coefficient of determination.
\end{abstract}

$\mathrm{K}^{\mathrm{N}}$ NOWLEDGE OF THE SPATIAL DISTRIBUTION in soil moisture content for the top 1 to $2 \mathrm{~m}$ of the earth's surface, known as the root zone, is important for understanding meteorologic, hydrologic, and agronomic processes. However, soil moisture is highly variable in space and time because of soil, topography, land cover, evapotranspiration, and precipitation heterogeneity (Engman, 1991; Wood et al., 1993). Furthermore, soil moisture prediction models are subject to representation, forcing, and parameterization error (Wood et al., 1993), and adequate spatial coverage by point measurements is prohibitive (Giacomelli et al., 1995) and soil moisture measurement from remote sensing observations is limited to the top few centimeters (Schmugge, 1985). As a result, research is being directed toward a combination of these three approaches for estimating the space and time variation of soil moisture content; assimilation of remote sensing observations into the soil moisture prediction model (e.g., Houser et al., 1998) and calibrationevaluation of the soil moisture model from point measurements (e.g., Walker, 1999). However, evaluation of such a system over even moderately sized areas is difficult because of a lack of knowledge about spatial variability in soil moisture and an inability to measure it at an appropriate scale within an adequate coverage. This necessitates a dependence on sparse point measurements of soil moisture and measurements of surrogate variables, such as surface temperature and evapotranspiration, for evaluation of the soil moisture estimates. To overcome such limitations, there is a demonstrated need for an instrument that allows rapid measurement

P.R. Houser, Hydrological Sciences Branch, Lab. for Hydrospheric Processes, NASA's Goddard Space Flight Center, Greenbelt, MD 20771; J.P. Walker, Dep. of Civil and Environmental Engineering, Univ. of Melbourne, Parkville, Victoria 3010, Australia. Received 15 Nov. 2000. *Corresponding author (j.walker@unimelb.edu.au).

Published in Soil Sci. Soc. Am. J. 66:728-734 (2002). of the spatial variability in soil moisture within the root zone.

Studies have shown that soil moisture content can be inferred from measurements of the soil electrical resistance (e.g., Seyfried, 1993; Amer et al., 1994; Hymer et al., 2000) by means of point measurement techniques with a coefficient of determination greater than 0.8 , when the pore water conductivity is low. A new instrument for mapping the spatial variability of electrical resistivity (resistance multiplied by a geometric factor) over the top several meters of the earth's surface is the OhmMapper by Geometrics Inc. (San Jose, CA). In this paper, we evaluate the OhmMapper's potential to map root zone soil moisture distribution over large areas in a timely fashion.

\section{MATERIALS AND METHODS}

\section{Study Area}

Evaluation of the OhmMapper was undertaken for a single 250-m transect running from top to bottom of a small watershed within the United States Department of Agriculture, Agricultural Research Center at Beltsville, MD, USA. The transect had an average terrain slope of $2 \%$ and consisted of a sandy loam soil ( $22 \%$ silt, $16 \%$ clay and $62 \%$ sand) with an average organic content of $3 \mathrm{~g} / \mathrm{cm}^{3}$.

Observations were made on a total of 8-d during a 30-d period immediately following corn (Zea mays L.) planting in June 2000 to capture a wide variation of soil moisture contents. The transect had very little plant biomass during the study period, ranging from initially bare fallow soil to a corn height of $15 \mathrm{~cm}$. Soil moisture was monitored every $25 \mathrm{~m}$ along the $250-\mathrm{m}$ transect to a depth of $70 \mathrm{~cm}$ by means of the widely accepted capacitance and time domain reflectometry point soil moisture measurement techniques. Continuous OhmMapper resistivity measurements were made along the entire length of the transect for 12 different configurations.

\section{Soil Moisture Measurements}

Point measurements of soil moisture content were made with the commercially available Theta Probe capacitance soil moisture instrument from Delta-T Devices Ltd. ${ }^{1}$ (Delta-T Devices Ltd., 1996) and the TRASE connector-type Time Domain Reflectometry (TDR) soil moisture instrument from Soil Moisture Equipment Corp. (Soil Moisture Equipment Corp., 1989). By inserting the portable soil moisture sensors vertically from the soil surface, the Theta Probe provided an average soil moisture measurement over the top $6 \mathrm{~cm}$ of soil, while the TDR instrument provided average soil moisture measurements over the top 15, 30, 45, 60 and $70 \mathrm{~cm}$ of soil, depending on the waveguide length used. The TDR waveguides with lengths greater than $15 \mathrm{~cm}$ were left in place between monitoring times, while shorter waveguides were reinserted for each measurement date. This was because the balun (connector between the waveguides and measurement device) could not

${ }^{1}$ The mention of trade and company names is for the benefit of the reader and does not imply an endorsement of the product. 

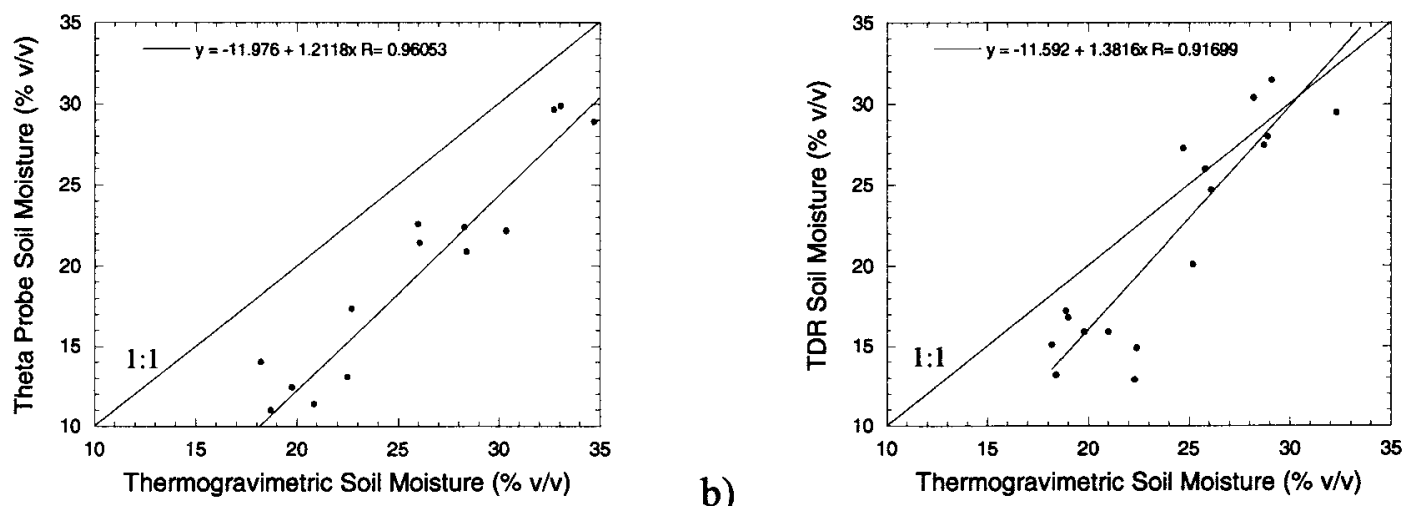

a)

b)

Fig. 1. Calibration of (a) 6-cm Theta Probe and (b) 15-cm TDR against thermogravimetric soil moisture measurements as compared to 1:1 line.

be attached to in situ probes of $15-\mathrm{cm}$ length without disturbing the installation; the Theta Probe waveguides were not detachable.

Comparison of 6-cm Theta Probe and 15-cm TDR soil moisture measurements with thermogravimetric (oven drying and weighing) soil moisture measurements showed that the manufacturers calibration was not satisfactory for an absolute determination of the soil moisture content, but there was a consistent linear relationship between the thermogravimetric measurements and the two electrical techniques used (Fig. 1). While an absolute soil moisture value was not required by this study, since we were primarily looking for a significant correlation between the resistivity measurements made by the OhmMapper and the point soil moisture measurements, the calibration correction was applied to the point measurements of soil moisture content. Although independent calibration was not performed for the longer TDR waveguides, because of the destructive nature of the test and the large number of samples required to make conclusive comments, we assumed that there would not be further accuracy loss for longer waveguides.

\section{Resistivity Measurements}

In conventional resistance surveys, four electrodes are inserted into the soil and a current injected into the ground by connecting a DC power source to two of the electrodes. The voltage is then measured at the remaining two electrodes, and the resistance calculated by Ohm's Law (Edwards, 1977). The resistance measured varies as a function of the distance and geometry between the probes, so it is normalized with a geometric correction factor that converts the measurement to resistivity (Geometrics Inc., 1999).

The OhmMapper instrument is a capacitively-coupled resistivity system that measures the electrical properties of the ground without the galvanic electrodes used in traditional resistivity surveys (Geometrics Inc., 1999). In this system, a coaxial-cable array with transmitter and receiver sections is pulled along the ground either by a single person or a small all-terrain vehicle (Fig. 2). This provides the instrument with a potential to cover large areas in a comparatively short period of time.

Like some configurations of traditional galvanic resistivity, the OhmMapper uses a dipole-dipole array (i.e., injection of current following measurement of voltage as opposed to a nested measurement) to measure resistivity, except that contact is made with the ground capacitively. The dipole-dipole array is very sensitive to horizontal changes in resistivity but relatively insensitive to vertical changes, meaning that it is good in mapping vertical structures but relatively poor in mapping horizontal structures (Loke, 1991).

By increasing the dipole cable length and/or tow-link length,

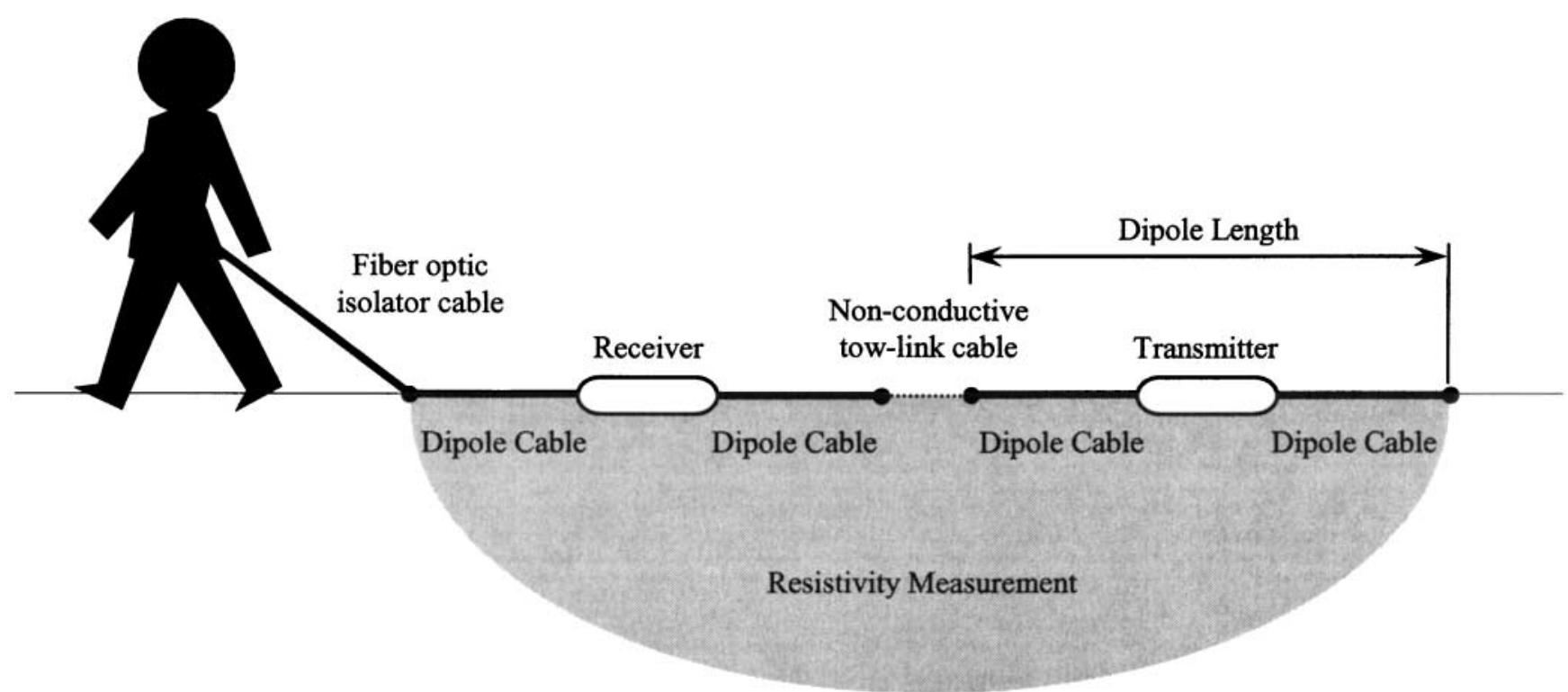

Fig. 2. Schematic of the OhmMapper instrument. 
and hence the distance between the midpoints of receiver and transmitter dipoles of a dipole-dipole array, the volume (having a half ellipsoid shape as indicated by Fig. 2), and hence depth of the resistivity measurement is increased. Various definitions exist for quantitatively describing this depth of investigation. Edwards (1977) has used the median depth of investigation (the depth at which $50 \%$ of the total response originates from above and $50 \%$ from below) to describe the depth of investigation while Roy and Apparao (1971) have used the depth at which the peak response occurs, which is typically at around two-thirds of the median depth. For dipoles separated by 0.2 and 0.5 fractions of a dipole length (see Fig. 2), the median depth of investigation is reported to be approximately 6 and $10 \%$ of the total length of the dipoledipole array respectively, and increases to approximately 14 and $22 \%$ of the total length of the dipole-dipole array for dipoles separated by one and six dipole lengths, respectively (Edwards, 1977). Intermediary distances of dipole separation have measurement depths corresponding to intermediary fractions of the total length of the dipole-dipole array. All dipole separations used in this study range from 0.05 to 0.8 of a dipole length, meaning that the median depth of investigation is expected to range from less than $6 \%$ to less than $14 \%$ of the total length of the dipole-dipole array.

Fink (1989), through reformulation of the work by Roy and Apparao (1971), has described the dipole-dipole array peak response depth of investigation $Z$ analytically by a simple power function

$$
Z=0.285 \cdot l \cdot n^{0.87},
$$

where $l$ is the dipole length and $n$ is the number of dipole lengths (or fraction thereof) between the dipoles.

By making multiple passes with different configurations (i.e., different dipole cable lengths and/or tow-link cable lengths) of the OhmMapper, it is possible to measure the variation of resistivity with depth. Nominal dipole cable lengths of $1,2.5$, and $5 \mathrm{~m}$, and nominal tow-link cable lengths of $0.25,0.5,1.0$ 2.0 and $4.0 \mathrm{~m}$, were used in this evaluation. The corresponding depth of investigation, as defined by Eq. [1], is given in Table 1 for the OhmMapper configurations used in this study. However, one must keep in mind that this relationship, like all electrical geophysical theory, has been derived for a homogeneous isotropic half-space, by means of traditional galvanic electrode arrangements and not a capacitively-coupled resistivity system. Moreover, the relationships for investigation depth do not represent the total depth over which the resistivity measurement is made, but rather about half of that, and the measured response is not uniform over that depth as in the case of TDR.

\section{THEORY}

Hymer et al. (2000) have used a power relationship to correlate volumetric soil moisture content $(\theta)$ and resistance $(R)$ of the form

$$
\theta=a \cdot R^{b},
$$

where $a$ and $b$ are fitted parameters. A relationship of this form is compatible with Archie's Law (Archie, 1942), where $a$ is the inverse of soil salinity and $b$ is a soil texture parameter. Similar non-linear relationships have also been used by Seyfried (1993) and Amer et al. (1994).

In this study, we take the OhmMapper apparent resistivity measurement to be equivalent to the resistance value in Eq. [2]. This is appropriate because conversion from resistivity to resistance is by multiplication with a geometric factor for the respective OhmMapper configuration, and we solve for the $a$ and $b$ parameters by fitting Eq. [2] to measurements of apparent resistivity and soil moisture independently for the different OhmMapper configurations. While resistivity measurements are sensitive to soil temperature, no correction has been applied to the measurements here. Our reasons for this are (i) by making OhmMapper observations in the early morning, soil temperature differences for the corresponding configurations were small and (ii) because soil temperature information can not be collected consecutively with the OhmMapper resistivity measurements, temperature corrections would not typically be made in routine OhmMapper applications.

In this analysis, the point soil moisture measurement at each $25-\mathrm{m}$ interval along the $250-\mathrm{m}$ transect is compared with the resistivity measurement centered on the point measurement. A shortcoming of this comparison is the assumption that the volume of point soil moisture measurements (approximately $5 \times 10^{2}$ to $5 \times 10^{3} \mathrm{~cm}^{3}$ ) is a representative sample of the moisture content in the soil volume attributing to the resistivity measurement (approximately $5 \times 10^{4}$ to $5 \times 10^{7} \mathrm{~cm}^{3}$ ). Moreover, the resistivity measurement is an average value over the length of the dipole-dipole array ( 5 to $25 \mathrm{~m}$ in our study) with varying depth and width along the length of the dipole-dipole array. This problem is not particular to this study; this is the case when evaluating any areal averaged estimate of soil moisture (such as from a soil moisture model or remote sensor) point measurements.

Although the variation of resistivity with depth in the soil profile could be evaluated from an inversion of multiple OhmMapper measurements with different dipole cable and towlink lengths using a commercial inversion software package, we have concentrated our efforts on evaluating the single pass resistivity data. Our reasons for this are (i) the point

Table 1. Comparison of coefficient of determination for the relationship between OhmMapper resistivity and soil moisture measurements for nominal dipole cable, tow-link, and soil moisture measurement depths given. The depth of investigation is that given by Eq. [1].

\begin{tabular}{|c|c|c|c|c|c|c|c|c|}
\hline \multirow{2}{*}{$\begin{array}{l}\text { Dipole cable } \\
\text { length }\end{array}$} & \multirow{2}{*}{$\begin{array}{c}\text { Tow-link } \\
\text { length }\end{array}$} & \multirow{2}{*}{$\begin{array}{l}\text { Depth of } \\
\text { investig. }\end{array}$} & \multicolumn{6}{|c|}{$R^{2}$-Value for soil moisture measurement depth } \\
\hline & & & $6 \mathrm{~cm}$ & $15 \mathrm{~cm}$ & $30 \mathrm{~cm}$ & $45 \mathrm{~cm}$ & $60 \mathrm{~cm}$ & $70 \mathrm{~cm}$ \\
\hline \multirow[t]{4}{*}{$1.0 \mathrm{~m}$} & $0.25 \mathrm{~m}$ & $10 \mathrm{~cm}$ & 0.004 & 0.049 & 0.028 & 0.028 & 0.016 & 0.007 \\
\hline & $0.50 \mathrm{~m}$ & $18 \mathrm{~cm}$ & 0.011 & 0.034 & 0.074 & 0.071 & 0.035 & 0.027 \\
\hline & $1.00 \mathrm{~m}$ & $32 \mathrm{~cm}$ & 0.000 & 0.020 & 0.030 & 0.031 & 0.058 & 0.016 \\
\hline & $2.00 \mathrm{~m}$ & $62 \mathrm{~cm}$ & 0.063 & 0.113 & 0.096 & 0.111 & 0.101 & $\underline{0.121}$ \\
\hline \multirow[t]{4}{*}{$2.5 \mathrm{~m}$} & $0.25 \mathrm{~m}$ & $12 \mathrm{~cm}$ & 0.048 & $\underline{0.130}$ & 0.104 & 0.110 & 0.064 & 0.089 \\
\hline & $0.50 \mathrm{~m}$ & $21 \mathrm{~cm}$ & 0.005 & 0.032 & $\mathbf{0 . 0 3 2}$ & 0.061 & $\underline{0.082}$ & $\mathbf{0 . 0 5 7}$ \\
\hline & $1.00 \mathrm{~m}$ & $38 \mathrm{~cm}$ & 0.262 & 0.310 & 0.240 & 0.298 & $\overline{0.342}$ & 0.266 \\
\hline & $2.00 \mathrm{~m}$ & $70 \mathrm{~cm}$ & 0.000 & 0.045 & 0.028 & $\underline{0.038}$ & $\overline{0.035}$ & 0.034 \\
\hline \multirow[t]{4}{*}{$5.0 \mathrm{~m}$} & $0.50 \mathrm{~m}$ & $22 \mathrm{~cm}$ & 0.048 & 0.183 & 0.218 & 0.161 & 0.141 & 0.104 \\
\hline & $1.00 \mathrm{~m}$ & $40 \mathrm{~cm}$ & 0.000 & 0.010 & 0.015 & 0.017 & 0.004 & $\underline{0.023}$ \\
\hline & $2.00 \mathrm{~m}$ & $74 \mathrm{~cm}$ & 0.005 & 0.051 & 0.052 & 0.106 & 0.101 & 0.130 \\
\hline & $4.00 \mathrm{~m}$ & $134 \mathrm{~cm}$ & 0.056 & 0.011 & 0.021 & 0.038 & 0.052 & 0.072 \\
\hline
\end{tabular}
Maximum $\boldsymbol{R}^{2}$-value for dipole cable and tow-link length configuration is underscored. 
measurements of soil moisture used in this evaluation give a depth integrated measurement of soil moisture content and (ii) the single pass data must be shown to have information about soil moisture before attempting the retrieval of soil moisture variation with depth. If the single pass resistivity data cannot be shown to have significant correlation with the average soil moisture content in the root zone, then it is highly unlikely that one will be able to retrieve the depth variation of soil moisture content in the root zone when using the multiple pass data and inversion software.

\section{RESULTS AND DISCUSSION}

The results from least squares fitting of the relationship given in Eq. [2] to the measured soil moisture content and resistivity data (see Fig. 3-5) are summarized in Table 1 by the coefficient of determination. These results show a typically low coefficient of determination, having a value of less than 0.4 for all dipole cable, towlink, and soil moisture depth measurement combinations investigated. A possible explanation for the apparently poor coefficient of determination found by this study, as compared with those found in previous studies on soil moisture-resistivity relationships, may be complicating effects of spatial variations in pore water conductivity, soil type, and soil temperature that have not been accounted for directly in this analysis. Moreover, this analysis assumes that the measured resistivity is attributed to a uniform response over a prescribed depth,

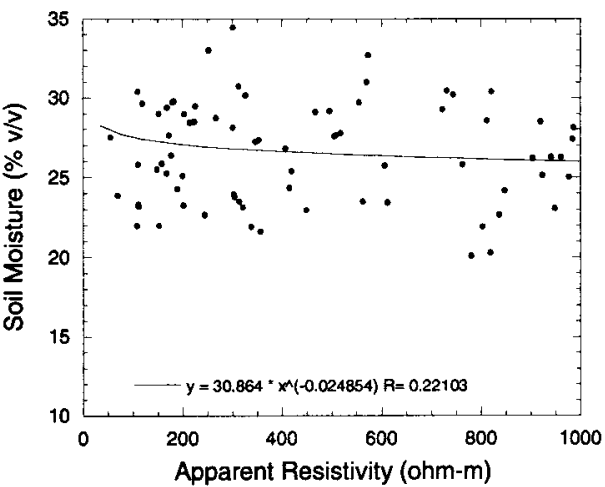

a)

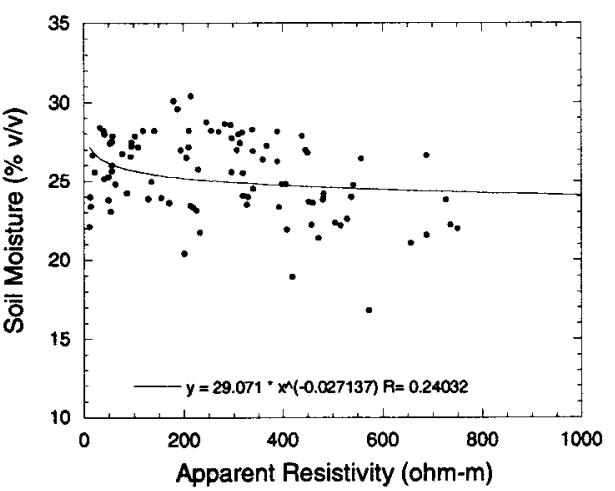

and that a single point measurement of soil moisture content is sufficient to describe the mean soil moisture content within the volume of soil contributing to the OhmMapper's measured response.

Generally speaking, for a given dipole cable length the soil moisture depth yielding the greatest coefficient of determination increased with tow-link length. The coefficients of determination ceased to follow this trend only when the soil moisture measurements used in the evaluation were apparently too shallow to capture the sampling depth of the resistivity measurements (i.e., 2.5-m dipole cable length with 2-m tow-link length and 5-m dipole cable length with greater than 1-m tow-link length). This trend was expected, as a greater separation between the midpoints of receiver and transmitter dipoles yields a greater depth of investigation, as given by Eq. [1] in Table 1. While the soil moisture measurement depth having the greatest coefficient of determination for a given OhmMapper configuration agrees closely with the suggested depth of investigation in some instances, it can be seen that the soil moisture measurement depth having the greatest coefficient of determination is typically greater than the suggested depth of investigation. This is not surprising for the reasons already stated in the previous discussion on depth of investigation.

We suggest that the lower coefficients of determination in Table 1 for shorter dipole cables and tow-link lengths are largely a result of the noisy resistivity mea-

b)
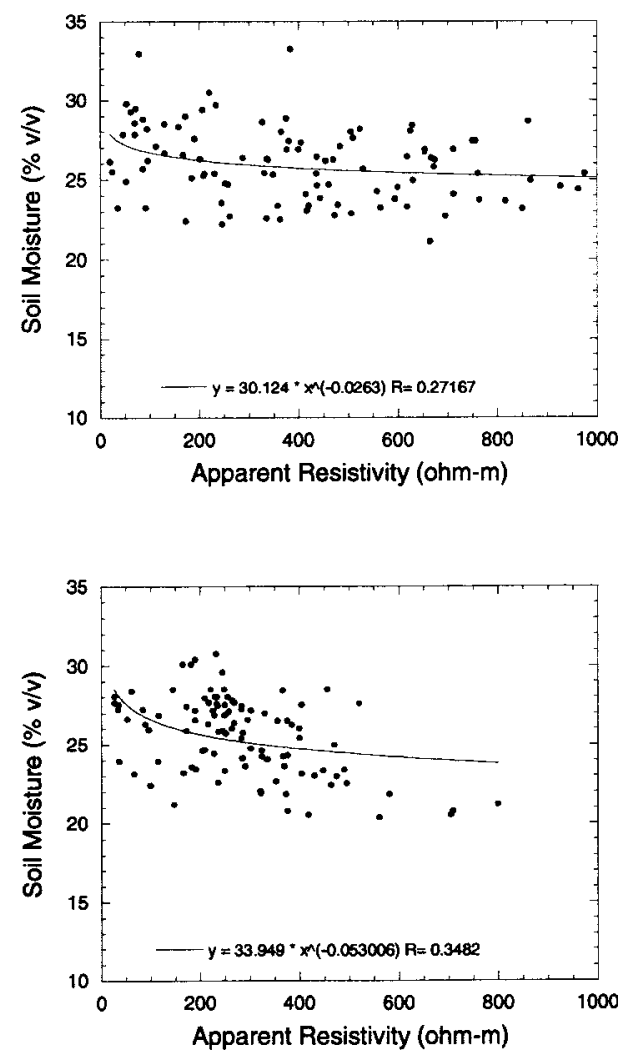

Fig. 3. Scatter plots of apparent resistivity and soil moisture measurements with fitted relationship, for the moisture measurement depth yielding the greatest coefficient of determination for the given OhmMapper configuration of dipole cable and tow-link lengths. Nominal 1-m dipole cable length with (a) nominal $0.25-\mathrm{m}$ tow-link length against 15-cm point measurements; (b) nominal 0.5 -m tow-link length against 30-cm point measurements; (c) nominal 1-m tow-link length against 60-cm point measurements; and (d) nominal 2-m tow-link length against 70-cm point measurements. 
a)
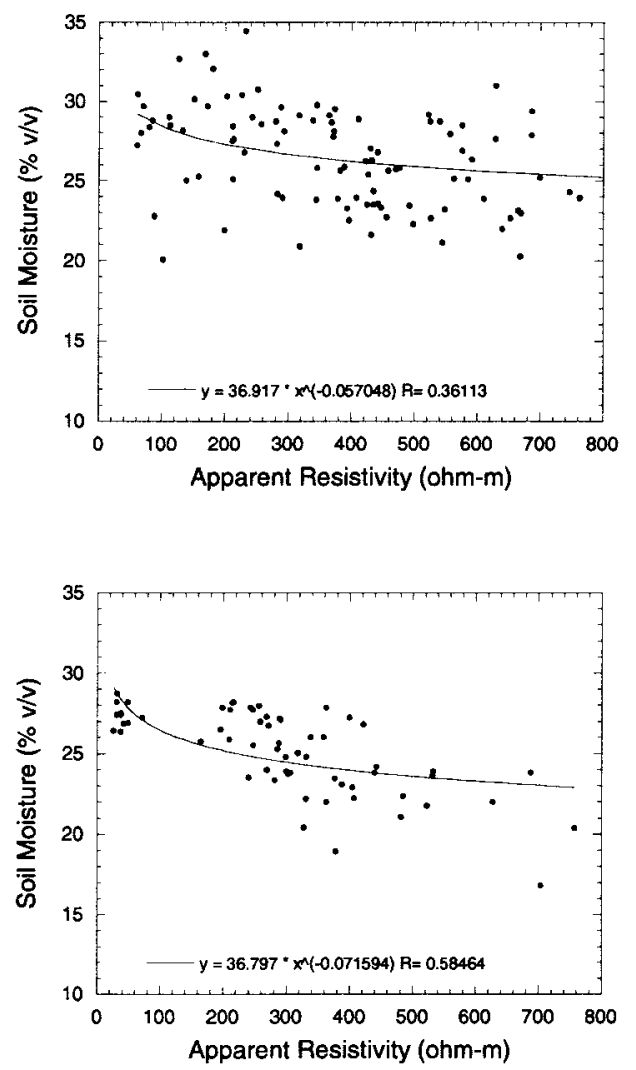

b)
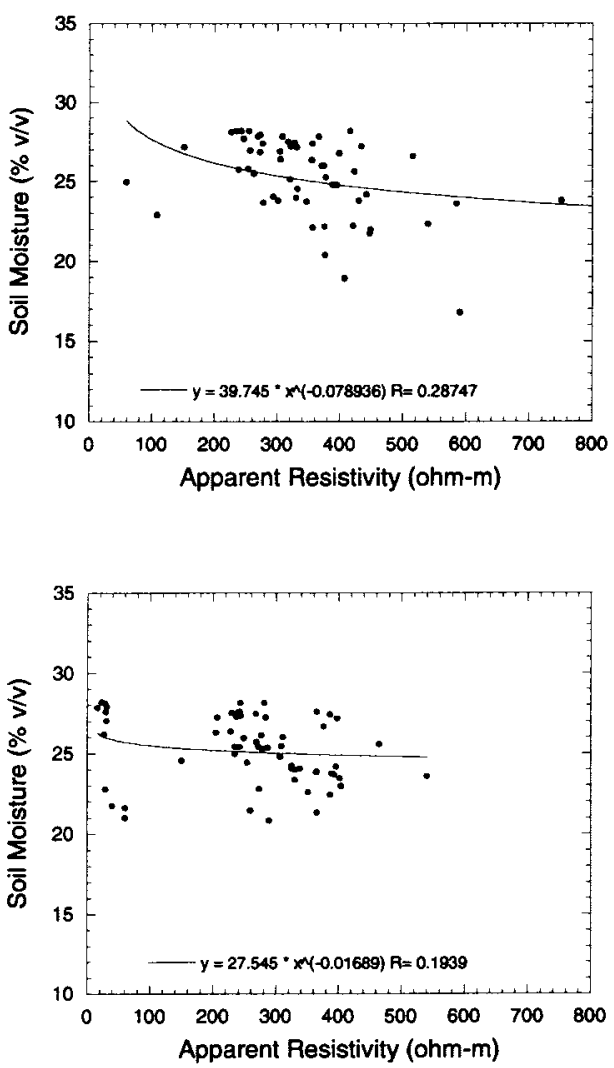

Fig. 4. As for Fig. 3 but nominal 2.5 -m dipole cable length with (a) nominal $0.25-\mathrm{m}$ tow-link length against 15-cm point measurements; (b) nominal $0.5-\mathrm{m}$ tow-link length against $60-\mathrm{cm}$ point measurements; (c) nominal 1-m tow-link length against 60 -cm point measurements; and (d) nominal 2-m tow-link length against $45-\mathrm{cm}$ point measurements.

surements yielded by the OhmMapper when the dipole cables were short and/or the dipole separation was small relative to the dipole length. While the longer dipole cables and/or tow-link lengths yielded less noisy measurements of resistivity, the lower coefficients of determination for the longest dipole cable and tow-link lengths are likely a result of the OhmMapper resistivity measurement being for a depth much greater than the deepest soil moisture measurement.

The OhmMapper configuration with 2.5-m dipole cable lengths and 1-m tow-link length yielded consistently higher coefficients of determination for all soil moisture measurement depths when compared with the other configurations (Table 1 ). This is likely a result of the combined effects of reduced noise in the OhmMapper resistivity measurements for this configuration, a substantial amount of the OhmMapper resistivity response originating from within the top $70 \mathrm{~cm}$ for this configuration, and a high correlation in the average soil moisture content over the various soil depths.

Scatterplots of soil moisture and apparent resistivity with the fitted relationships are given in Fig. 3 to 5 for each dipole cable and tow-link length for the soil moisture measurement depth having the greatest coefficient of determination from Table 1 . However, it may be seen that there is as much as $15 \%(\mathrm{v} / \mathrm{v})$ variation in soil moisture content for a given resistivity value, with most scatterplots bearing little or no resemblance to the fitted relationship from Eq. [2], or any other relationship one might wish to apply, being simply a cloud of points. The nominal 2.5-m dipole cable length with 1-m tow-link cable length (Fig. 4c) is the only OhmMapper configuration that yielded a scatterplot bearing significant resemblance to the fitted relationship, as indicated by its higher coefficient of determination (Table 1 ).

Soil moisture variation along the $250-\mathrm{m}$ transect is plotted in Fig. 6 for the 60 -cm-deep soil moisture point measurements, and is compared with the inferred (by means of the fitted relationship from Fig. 4c) soil moisture from OhmMapper measurements with 2.5-m dipole cables and 1-m tow-link lengths. This is the combination of soil moisture measurement depth and OhmMapper configuration that yielded the greatest coefficient of determination in Table 1. Most notable in this figure is that point measurements of soil moisture content have a variation of approximately $7 \%(\mathrm{v} / \mathrm{v})$ from dry to wet, while the inferred OhmMapper soil moisture has only approximately $3 \%(\mathrm{v} / \mathrm{v})$ variation from dry to wet. However, the day on which point measurements indicate the wettest soil moisture coincides with the day on which OhmMapper measurements indicate the wettest soil moisture. Likewise, the point soil moisture and OhmMapper data both indicate the driest soil moisture on the same day. Moreover, the inferred OhmMapper soil moisture exhibits approximately the correct trend along the transect when compared with the point soil moisture measurements. Hence, with appropriate scaling knowledge, one may be able to develop a much better fit to 
a)

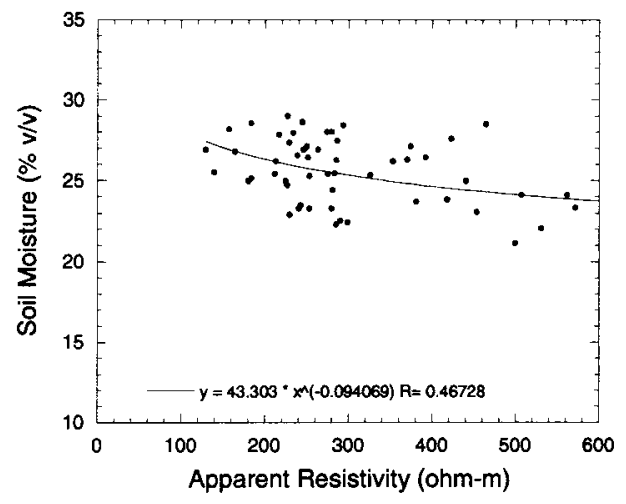

c)

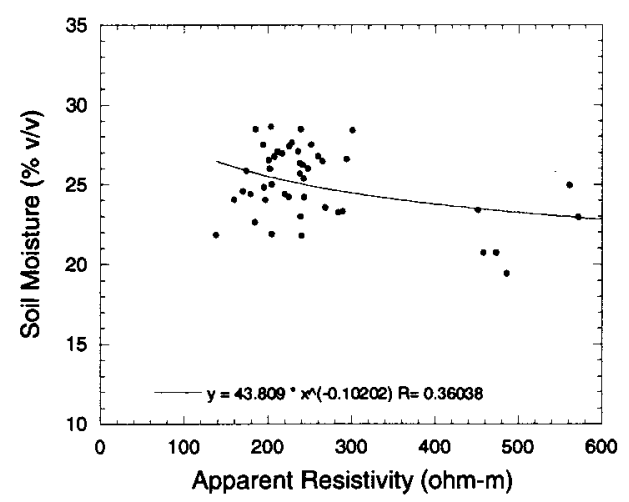

b)

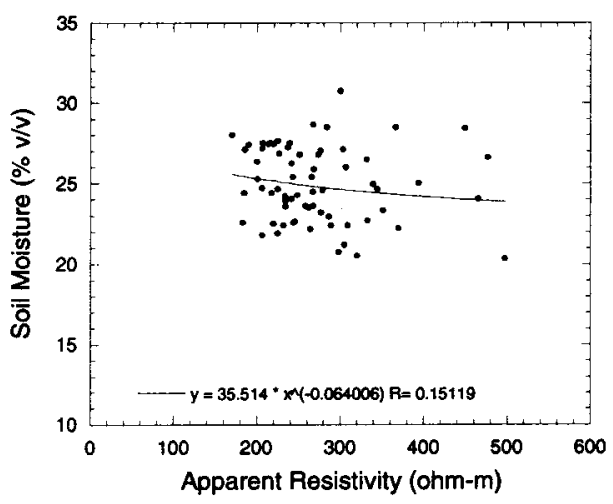

d)

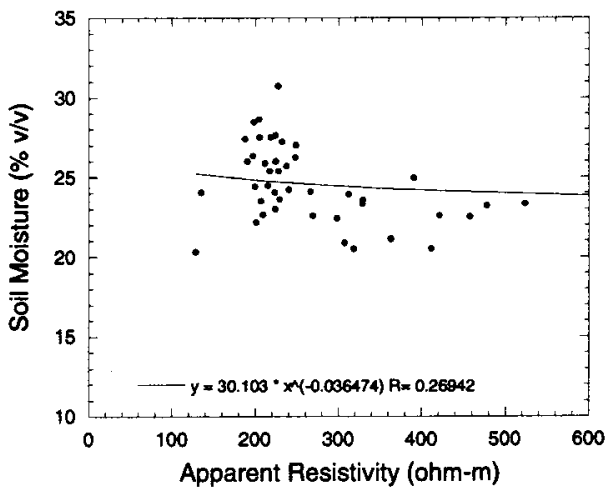

Fig. 5. As for Fig. 3 but nominal 5-m dipole cable length with (a) nominal 0.5-m tow-link length against 30-cm point measurements; (b) nominal 1-m tow-link length against 70-cm point measurements; (c) nominal 2-m tow-link length against 70-cm point measurements; and (d) nominal 4-m tow-link length against 70-cm point measurements.

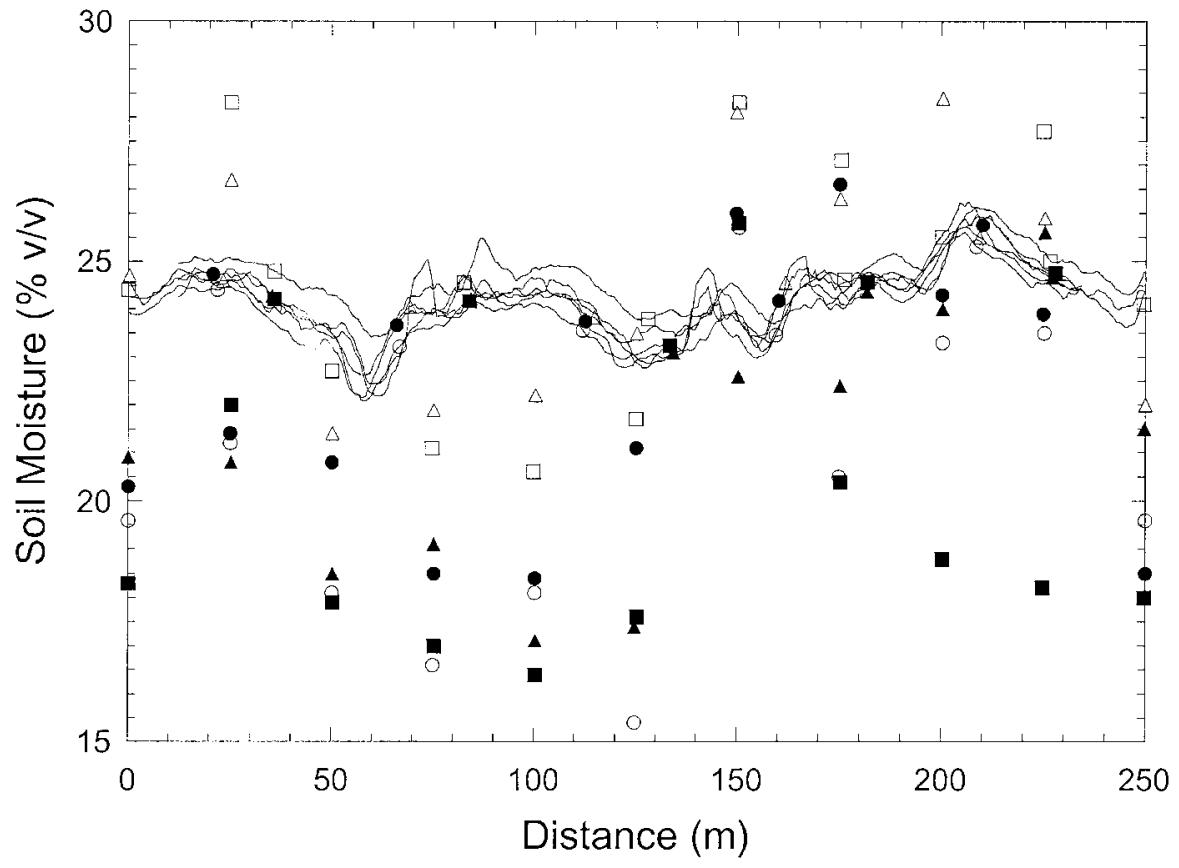

Fig. 6. Comparison of $60-\mathrm{cm}$ point soil moisture measurements (symbols) with the inferred OhmMapper soil moisture (continuous line and symbol) from the $2.5-\mathrm{m}$ dipole cable length and 1-m tow-link length configuration; the different symbols correspond to the different dates for which measurements were made. 
the point measurements of soil moisture content along the 250-m transect.

It is well known that the near-surface soil moisture content responds rapidly to both infiltration and exfiltration events while the soil moisture content for a deep layer of soil has a much slower response time. Moreover, changes in the near-surface (i.e., $0.5 \mathrm{~m}$ ) soil moisture content have only a minimal impact on the average soil moisture content of a thick soil layer (i.e., $3 \mathrm{~m}$ ). Hence, an explanation for the OhmMapper having the correct response to soil moisture content but the wrong magnitude may be that the OhmMapper resistivity measurements are indeed responding to the soil moisture content of a much deeper layer than that measured by the point measurements, as suggested already in the foregoing discussion. Furthermore, OhmMapper measurements of resistivity are an average measurement made not only over some depth, but over the entire length of the instrument (i.e., $11 \mathrm{~m}$ for 2.5-m dipole cables and 1-m tow-link), meaning that interpretation of comparisons with individual point measurement is difficult. The problem of disparity in measurement scales has already been discussed in the previous section.

\section{CONCLUSIONS}

This study has shown a coefficient of determination of approximately 0.35 when fitting a power law relationship to the time domain reflectometry point measurements of soil moisture content and the OhmMapper measurements of apparent resistivity with $2.5-\mathrm{m}$ dipole cables and a 1-m tow-link. All other OhmMapper configurations resulted in lower coefficients of determination for the soil moisture depths measured. Results have indicated that this configuration is optimal for the purpose of measuring soil moisture in the root zone, with the coefficient of determination likely to be greater if comparison measurements of soil moisture content are made for a greater depth and/or a more sophisticated analysis of the resistivity measurements is undertaken. Moreover, unless the noise in OhmMapper measurements of resistivity can be reduced for shorter dipole cables and/or shorter dipole cable separations, it is unlikely that the OhmMapper will be applicable for measuring the soil moisture content of shallower soil depths or be used to measure the variation of soil moisture content with depth in the root zone. However, a single pass with the OhmMapper instrument (2.5-m dipole cables and 1-m tow link) may be an appropriate means for providing an estimate of the average soil moisture content in the top few meters.

\section{ACKNOWLEDGMENTS}

The USDA-ARS is acknowledged for allowing access to the field site located at the Agriculture Research Center in Beltsville, MD. Assistance was given in the field by Kristi Arsenault, Brian Cosgrove, Dan Hymer, and Melinda Pouss. The loan of an OhmMapper by Geometrics Inc. and preliminary analysis of OhmMapper data by Brandy Rutledge is acknowledged.

\section{REFERENCES}

Amer, S.A., T.O. Keefer, M.A. Weltz, D.C. Goodrich, and L.B. Bach, 1994. Soil moisture sensors for continuous monitoring. Water Resour. Bull. 30:69-83.

Archie, G.E. 1942. The electrical resistivity $\log$ as an aid in determining some reservoir characteristics. Trans. Am. Inst. Min. Eng. 146:5462.

Delta-T Devices Ltd. 1996. Theta Probe Soil Moisture Sensor Type ML1 User Manual. Delta-T Devices, Inc., Cambridge, England.

Edwards, L.S. 1977. A modified pseudosection for resistivity and induced-polarization. Geophysics 42:1020-1036.

Engman, E.T. 1991. Application of microwave remote sensing of soil moisture for water resources and agriculture. Remote Sens. Environ. 35:213-226.

Fink, J.B. 1989. Induced polarization: electrochemistry, fractal geometry, and geohydrological applications. Ph.D. dissertation (Diss. Abstr. Micro- fiche E9791 1989 669), Univ. of Arizona, Tucson.

Geometrics. 1999. OhmMapper TR1 Operation Manual. Geometrics Inc., San Jose, CA.

Giacomelli, A., U. Bacchiega, P.A. Troch, and M. Mancini, 1995. Evaluation of surface soil moisture distribution by means of SAR remote sensing techniques and conceptual hydrological modelling. J. Hydrol. 166:445-459.

Houser, P.R., W.J. Shuttleworth, J.S. Famiglietti, H.V. Gupta, K.H. Syed, and D.C. Goodrich. 1998. Integration of soil moisture remote sensing and hydrologic modeling using data assimilation. Water Resour. Res. 34:3405-3420.

Hymer, D.C., M.S. Moran, and T.O. Keefer. 2000. Soil water evaluation using a hydrologic model and calibrated sensor network. Soil Sci. Soc. Am. J. 64:319-326.

Loke, M.H. 1999. Electrical imaging surveys for environmental and engineering studies: A practical guide to 2D and 3D surveys. Geometrics, San Jose, CA

Roy, A., and A. Apparao. 1971. Depth of investigation of direct current methods. Geophysics 36:943-959.

Schmugge, T. 1985. Remote sensing of soil moisture. p. 101-124. In M.G. Anderson and T.P. Burt (ed.) Hydrological forecasting. John Wiley and Sons, New York.

Seyfried, M.S. 1993. Field calibration and monitoring of soil-water content with fiberglass electrical resistance sensors. Soil Sci. Soc. Am. J. 57:1432-1436.

Soil Moisture Equipment Corp. 1989. Trase System 1 Operating Instructions. Soil Moisture Equipment Corp., Santa Barbara, CA.

Walker, J.P. 1999. Estimating soil moisture profile dynamics from near-surface soil moisture measurements and standard meterological data. PhD. Dis., Dep. of Civil, Surveying, and Environmental Engineering, University of Newcastle, Australia.

Wood, E.F., D.S. Lin, M. Mancini, D.J. Thongs, P.A. Troch, T.J Jackson, J.S. Famiglietti, and E.T. Engman. 1993. Intercomparisons between passive and active microwave remote sensing and hydrological modeling for soil moisture. Adv. Space Res. 13:167-176. 